\title{
Mobile Phones and Head Tumours: A Critical Analysis of Case-Control Epidemiological Studies
}

\author{
Angelo Gino Levis ${ }^{1}$, Nadia Minicuci ${ }^{2}$, Paolo Ricci ${ }^{3}$, Valerio Gennaro ${ }^{4}$ and Spiridione Garbisa ${ }^{*}, 1$ \\ ${ }^{I}$ Department of Experimental Biomedical Sciences, Medical School of Padova, Padova, Italy \\ ${ }^{2}$ Institute of Neuroscience, National Research Council, Padova, Italy \\ ${ }^{3}$ Local Health Unit, Mantova, Italy \\ ${ }^{4}$ National Cancer Research Institute (IST), Genova, Italy
}

\begin{abstract}
Protocols and results of case-control studies, pooled- and meta-analyses on the relationship between mobile phone use and risk of head tumours are critically analysed, and for each study strict elements necessary for evaluating its reliability are identified and applied (see Methods). In studies funded by public bodies, blind protocols give positive results revealing cause-effect relationships between long-term latency or use of mobile phones (cellulars and cordless) and statistically significant increases of ipsilateral risk of brain gliomas and acoustic neuromas, with biological plausibility. In studies funded or co-funded by the cellphone companies non-blind protocols give overall negative results with systematic underestimation of risk; however, also in these studies a statistically significant increase in risk of ipsilateral brain gliomas, acoustic neuromas, and parotid gland tumours is quite common when only subjects with at least 10 years of latency or exposure to mobile phones (only cellulars) are considered. Informed precautionary measures are therefore recommended as a step towards reducing risk of head tumours, especially for young people and those making intense use of cellular and cordless phones.
\end{abstract}

Keywords: Cellular and cordless phones; head tumours; epidemiological case-control studies; bias, flaws and conflicts of interest.

\section{INTRODUCTION}

The worldwide spread of the use of mobile phones (MPs: analogue and digital cellulars, and cordless) has heightened concerns about possible adverse effects, especially head tumours. According to the International Telecommunications Union, the number of cellphone subscriptions has reached 5.5 billion (end of 2011), with over half of all users thought to be children or young adults. There are no data for cordless users - also exposed to radiofrequencies (rf) - but a figure of 2 billion is a reasonable assumption. Given these figures, even an established modest increase (20-30\%) in tumour risk for MP users would result in significant social and health costs and individual suffering, while higher risks could give rise to a health crisis of dramatic proportions.

MPs were introduced into the market in the 1980s, and widely used for the following decade in the USA, the Scandinavian countries and Israel. Since the beginning of the 1990s, MP use has become practically universal, with the consequence that there has been exposure to MP radiation throughout almost the entire world for at least 20 years. Although brain and cranial nerve tumours may have very long latency times (up to 30 years or more), it is likely that some due to MPs will be diagnosed after just 10-15 years of MP use or latency - as has been the case for long-latency

*Address correspondence to this author at the Department of Experimental Biomedical Sciences, Viale G. Colombo 3, 35121 Padova, Italy; Tel: +39 049 8276088; Fax: +39049 8276089; E-mail: garbisa@unipd.it tumours resulting from other hazards of recent times, namely ionizing radiations, asbestos and smoking.

Whether or not there is a relationship between MP use and head tumour risk (brain tumours, acoustic neuromas, and salivary gland tumours) is still under debate. On the one hand, there are the researchers who recognise the validity of positive results, and are recommending application of the "Precautionary Principle", especially for children who face decades of exposure - one such is Hardell [1-4], who has documented a statistically significant (s.s.) increase ( $\geq$ $100 \%$ ) of head tumours (brain gliomas and acoustic nerve neuromas) in people with MP exposure or latency of at least 10 years. But on the other hand there are researchers who reach their conclusions, largely reassuring, on the basis of the results of the Interphone project, which involved research groups from 13 countries [5, 6] and - according to their interpretation - failed to find any increased risk of head tumours in MP users.

In view of the possible implications for human health, it is therefore vital to understand the reasons behind the conflict between Hardell's positive results and those from Interphone and other studies. Progress requires a critical analysis of the methodological elements necessary for an impartial evaluation of contradictory studies.

\section{METHODS}

We have carried out a critical examination of the protocols, results and interpretations from all case-control studies, pooled-analyses and meta-analyses on head tumour 
risk among MP users, and for each study have identified the elements that should be taken into account to ensure an impartial evaluation of its reliability, namely:

- $\quad$ the number of subjects selected (cases and controls), and the percentage of their participation in the study;

- $\quad$ the percentage of actually exposed subjects, based on the frequency and duration of the MP use;

- the inclusion among the exposed of all users of MPs, cordless included, as well as of the most exposed subjects - those making intense use of MPs, youngest and those living in rural areas;

- the compatibility of latency and/or exposure time since first use of MPs with the progression time of the examined tumours ( $\geq 10$ years);

- the laterality of the head tumour localisation relative to the habitual laterality of MP use;

- the distribution of the relative risk values ("Odds Ratios", OR) $>$ or $<1$, their statistical significance ( $95 \%$ Confidence Interval, CI), and the probability that such distribution might be random;

- the full and correct selection and citation of data included in the meta-analyses;

- the financial support by independent sources or by cellphone companies.

We have quantified the total number of OR values from each study, independently of sex, age, exposure time or latency of the examined subjects. Since the OR estimates reported by each author are not independent, a statistical comparison between the percentages of ORs $>1$ or $<1$ is difficult. However, a simple comparison of their percentage may indicate if their differences are more or less random, or might be due to a significantly increased risk or a substantial protective effect, or else - in the absence of plausibility of either of these effects - to errors and/or distortions in the study design.

\section{RESULTS}

The pooled analyses of epidemiological case-control studies by Hardell produced positive results indicating a cause-effect relationship: MP exposure for or latency of at least 10 years increased by up to $100 \%$ the risk of tumour ipsilateral with the side of the head preferred for phone use the only side significantly irradiated - with statistical significance for brain gliomas and acoustic neuromas [1-4]. On the contrary, studies published under the Interphone project produced "negative" results and are characterized by a substantial underestimation of the risk of tumour $[5,6]$.

The data published in 2010 by Interphone including the risk of malignant (gliomas) and benign (meningiomas) brain tumours [5], as well as those published in 2011 including the risk of acoustic neuromas [6] in people using only cellular phones (not cordless), have been widely publicised as reassuring by their authors as well as by the organisations that promoted and funded the study (International Agency for Research on Cancer - IARC - and European Union 70\%, the cellphone companies 30\%) [7], the main agencies responsible for protecting human health [8-13], and over 100 newspapers which printed reassuring headlines round the world. This is the case despite the first Interphone article [5] being accompanied by a commentary by Saracci and Samet with a very telling title: "Call me on my mobile phone...or better not? - a look at the INTERPHONE study results" [14]; they pointed out some major defects of the Interphone protocol and results which would have substantially "diluted" risk estimates:

- a too-short daily cellphone use (mean: 2-5 min a day), even in the highest-exposure category, relative to the intense use prevailing today;

- a too-short exposure or latency time: less than $5 \%$ of meningioma cases and less than $9 \%$ of glioma cases have at least 10 years of MP use or latency;

- $\quad$ the systematic reduction in risk (at least $30 \%$ ), which has a minimum probability of being due to chance so supporting the contention, without any biological plausibility, that cellphone use could provide protection from developing cancer.

The conclusions of Saracci and Samet were therefore that: "Overall, a consistent inter-country pattern of replicated reduced risk for both meningioma and glioma is shown ... with only three odds ratios (ORs) out of 50 above 1 . These results are also in line with the detailed findings already published in separate reports, involving nearly half of the cancer cases, from some of the participating countries. On the null hypothesis that there is no association between mobile phone use and brain cancer, ORs fluctuating randomly above and below 1 would be expected, whereas the observed patterns of reduced risks, on average of the order of $30 \%$, would have a tiny probability of occurring just by chance. Having ruled out chance and the possibility of a protective effect - absent any supporting biological evidence less plausible than the possibility of bias from a variety of sources, bias stands as the most likely explanation of the observed results. As already noted, this interpretation carries the uncomfortable consequence that the interpretation of any result of the study becomes problematic, unless the sources of bias are identified and their consequences quantified ... A substantiated downward and generalized bias implies that any observed increase in risk would be underestimated, independent of statistical significance ... Therefore we commend the Interphone investigators for a rigorously implemented protocol and the careful exploration of bias. Interphone clearly demonstrates that epidemiological research has to give major emphasis to bias prevention and control".

In this context, we consider extremely important the editorial by Cardis - former coordinator of the Interphone project itself - and Sadetzki [15]. This latter headed the Israeli Interphone team, and her own studies - showing large increases in parotid tumour risk in regular and long-time users of cellphones [16] - were presented in September 2009 to the US Senate [17]. Their editorial [15] has a rather eloquent title: "Indications of possible brain-tumour risk in mobile-phone studies: should we be concerned? ". Furthermore, the highly risk-assertive statements of the two authors were not based on new experimental data, but instead on a critical review of the results of the Interphone final study on brain tumour risk in cellphone users, to which they themselves contributed [5]. In our view their stance represents a milestone in the quest for truth. 
The editorial of Cardis and Sadetzki takes further the remarks by Saracci and Samet, and leaves little doubt about the relevance of our criticisms, which we document on as follows (Table 1):
- of the 17 Interphone studies [16, 18-33], fewer than $10 \%$ of the exposed cases and controls had completed at least 10 years of cellphone latency or continued use, which means that over $90 \%$ had an inadequate exposure time. Since in most of the tumours

Table 1. Main Features of the Case-Control Studies by Hardell and Interphone on the Relationships Between MP Use and Head Tumours

\begin{tabular}{|c|c|c|c|c|c|c|c|c|c|c|c|c|}
\hline \multirow{2}{*}{\multicolumn{2}{|c|}{ Author (Tumour Type) }} & \multirow{2}{*}{ Ref. } & \multicolumn{2}{|c|}{ n. and \% Participants } & \multicolumn{2}{|c|}{ n. and \% Exposed } & \multicolumn{2}{|c|}{$\begin{array}{c}\text { n. and \% Exposed } \\
\geq 10 \mathrm{y}\end{array}$} & \multicolumn{2}{|c|}{$\begin{array}{c}\text { n. and } \% \\
O R \neq 1\end{array}$} & \multirow{2}{*}{$\begin{array}{c}\text { n. and \% } \\
95 \% \text { CI }<1 \\
\text { Stat. Signif.(1) } \\
\text { on tot. OR }<1\end{array}$} & \multirow{2}{*}{$\begin{array}{c}\text { n. and \% } \\
95 \% \text { CI }>1 \\
\text { Stat. Signif.(1) } \\
\text { on tot. OR }>1\end{array}$} \\
\hline & & & Cases & Controls & Cases & Controls & Cases & Controls & $<1$ & $>1$ & & \\
\hline \multirow{6}{*}{$\begin{array}{l}\text { Hardell et al. } \\
\text { (benign head tumours) } \\
\text { Hardell } \text { et al. } \\
\text { (malign head tumours) } \\
\text { total }\end{array}$} & 2006 & [2] & 1254 & 2162 & 672 & 1172 & 98 & 147 & 20 & 282 & 0 & 101 \\
\hline & & & $88 \%$ & $89 \%$ & $54 \%$ & $54 \%$ & $8 \%$ & $7 \%$ & $7 \%$ & $93 \%$ & $0 \%$ & $36 \%$ \\
\hline & 2006 & {$[1]$} & 905 & 2162 & 583 & 1172 & 134 & 147 & 15 & 208 & 0 & 81 \\
\hline & & & $90 \%$ & $89 \%$ & $64 \%$ & $54 \%$ & $15 \%$ & $7 \%$ & $7 \%$ & $93 \%$ & $0 \%$ & $39 \%$ \\
\hline & & & 2159 & 4324 & 1255 & 1172 & 232 & 147 & 35 & 490 & 0 & 182 \\
\hline & & & $89 \%$ & $89 \%$ & $58 \%$ & $54 \%$ & $18 \%$ & $13 \%$ & $7 \%$ & $93 \%$ & $0 \%$ & $37 \%$ \\
\hline \multirow{2}{*}{$\begin{array}{l}\text { Christensen } \text { et al. } \\
\text { (acoustic neuromas) }\end{array}$} & 2004 (2) & [21] & 107 & 214 & 61 & 147 & 2 & 15 & 13 & 4 & 1 & 0 \\
\hline & & & $76 \%$ & $64 \%$ & $57 \%$ & $69 \%$ & $3 \%$ & $10 \%$ & $77 \%$ & $23 \%$ & $0,8 \%$ & $0 \%$ \\
\hline \multirow{2}{*}{$\begin{array}{l}\text { Lonn et al. } \\
\text { (acoustic neuromas) }\end{array}$} & 2004 & [29] & 148 & 604 & 89 & 356 & 14 & 29 & 16 & 16 & 0 & 2 \\
\hline & & & $93 \%$ & $72 \%$ & $60 \%$ & $59 \%$ & $16 \%$ & $8 \%$ & $50 \%$ & $50 \%$ & $0 \%$ & $12,5 \%$ \\
\hline \multirow{2}{*}{$\begin{array}{l}\text { Lonn et al. } \\
\text { (gliomas, meningiomas) }\end{array}$} & 2005 & [22] & 644 & 674 & 327 & 780 & 30 & 65 & 180 & 15 & 15 & 0 \\
\hline & & & $78 \%$ & $71 \%$ & $51 \%$ & $59 \%$ & $9 \%$ & $8 \%$ & $92 \%$ & $8 \%$ & $8,3 \%$ & $0 \%$ \\
\hline \multirow{2}{*}{$\begin{array}{l}\text { Christensen et al. } \\
\text { (gliomas, meningiomas) }\end{array}$} & 2005 & [23] & 427 & 822 & 244 & 560 & 20 & 39 & 40 & 11 & 4 & 0 \\
\hline & & & $61 \%$ & $64 \%$ & $48 \%$ & $56 \%$ & $8 \%$ & $7 \%$ & $78 \%$ & $22 \%$ & $10 \%$ & $0 \%$ \\
\hline \multirow{2}{*}{$\begin{array}{l}\text { Schoemaker et al. } \\
\text { (acoustic neuromas) }\end{array}$} & 2005 & [31] & 678 & 3553 & 360 & 1934 & 47 & 212 & 35 & 17 & 1 & 1 \\
\hline & & & $82 \%$ & $42 \%$ & $53 \%$ & $55 \%$ & $13 \%$ & $11 \%$ & $67 \%$ & $33 \%$ & $2,8 \%$ & $5,9 \%$ \\
\hline \multirow{2}{*}{$\begin{array}{l}\text { Lahkola et al. } \\
\text { (head tumours) }\end{array}$} & 2005 & [30] & 726 & 456 & n.r. & n.r. & n.r. & n.r. & 7 & 0 & 5 & 0 \\
\hline & & & $88 \%$ & $42 \%$ & n.r. & n.r. & n.r. & n.r. & $100 \%$ & $0 \%$ & $71 \%$ & $0 \%$ \\
\hline \multirow{2}{*}{$\begin{array}{l}\text { Takebayashi et al. } \\
\text { (acoustic neuromas) }\end{array}$} & 2006 & [18] & 97 & 330 & 51 & 192 & 0 & 0 & 9 & 2 & 0 & 0 \\
\hline & & & $81 \%$ & $51 \%$ & $53 \%$ & $58 \%$ & $0 \%$ & $0 \%$ & $82 \%$ & $18 \%$ & $0 \%$ & $0 \%$ \\
\hline \multirow{2}{*}{$\begin{array}{l}\text { Hepworth et al. } \\
\text { (gliomas) }\end{array}$} & 2006 & [24] & 966 & 1716 & 505 & 896 & 48 & 67 & 31 & 6 & 2 & 1 \\
\hline & & & $51 \%$ & $45 \%$ & $52 \%$ & $52 \%$ & $10 \%$ & $7 \%$ & $84 \%$ & $16 \%$ & $6,4 \%$ & $16,7 \%$ \\
\hline \multirow{2}{*}{$\begin{array}{l}\text { Schuz et al. } \\
\text { (gliomas, meningiomas) }\end{array}$} & 2006 & [25] & 747 & 1494 & 229 & 492 & 17 & 20 & 44 & 10 & 1 & 0 \\
\hline & & & $84 \%$ & $61 \%$ & $31 \%$ & $33 \%$ & $7 \%$ & $4 \%$ & $81 \%$ & $19 \%$ & $2,3 \%$ & $0 \%$ \\
\hline \multirow{2}{*}{$\begin{array}{l}\text { Lonn et al. } \\
\text { (parotid gland tumours) }\end{array}$} & 2006 (2) & [26] & 172 & 681 & 101 & 587 & 6 & 51 & 32 & 15 & 0 & 0 \\
\hline & & & $87 \%$ & $70 \%$ & $59 \%$ & $60 \%$ & $6 \%$ & $9 \%$ & $68 \%$ & $32 \%$ & $0 \%$ & $0 \%$ \\
\hline Klaeboe et al. & 2007 & [19] & 541 & 358 & 279 & 675 & 0 & 0 & 92 & 11 & 9 & 0 \\
\hline \multicolumn{3}{|c|}{ (acoustic neuromas, gliomas, meningiomas) } & $74 \%$ & $69 \%$ & $52 \%$ & $50 \%$ & $0 \%$ & $0 \%$ & $89 \%$ & $11 \%$ & $14,7 \%$ & $0 \%$ \\
\hline Lahkola et al. & 2007 & [32] & 1521 & 3301 & 869 & 1854 & 143 & 220 & 67 & 16 & 37 & 1 \\
\hline (gliomas) & & & $60 \%$ & $50 \%$ & $58 \%$ & $59 \%$ & $16 \%$ & $12 \%$ & $81 \%$ & $19 \%$ & $55 \%$ & $6,3 \%$ \\
\hline Hours et al. & 2007 & [27] & 350 & 455 & 188 & 257 & 3 & 1 & 69 & 44 & 2 & 0 \\
\hline (acoustic neuromas, gliom & $\mathrm{s}$, meningic & mas) & $71 \%$ & $71 \%$ & $54 \%$ & $56 \%$ & $2 \%$ & $0,4 \%$ & $61 \%$ & $39 \%$ & $2,9 \%$ & $0 \%$ \\
\hline Schlehofer et al. & 2007 (2) & [20] & 97 & 194 & 28 & 73 & 0 & 3 & 8 & 2 & 1 & 0 \\
\hline (acoustic neuromas) & & & $89 \%$ & $53 \%$ & $29 \%$ & $38 \%$ & $0 \%$ & $4 \%$ & $80 \%$ & $20 \%$ & $12,5 \%$ & $0 \%$ \\
\hline Sadetzki et al. & 2007 & [16] & 460 & 1266 & 570 & 1372 & 0 & 0 & 75 & 63 & 0 & 6 \\
\hline (salivary and parotid glanc & tumours) & & $87 \%$ & $66 \%$ & $62 \%$ & $54 \%$ & $0 \%$ & $0 \%$ & $54 \%$ & $46 \%$ & $0 \%$ & $10 \%$ \\
\hline Lahkola et al. & 2008 & [33] & 1209 & 3299 & 573 & 1696 & 73 & 212 & 62 & 2 & 27 & 0 \\
\hline (meningiomas) & & & $74 \%$ & $50 \%$ & $47 \%$ & $58 \%$ & $13 \%$ & $13 \%$ & $97 \%$ & $3 \%$ & $43,5 \%$ & $0 \%$ \\
\hline Takebayashi et al. & 2008 & [28] & 322 & 683 & 173 & 329 & 11 & 10 & 49 & 33 & 0 & 0 \\
\hline (gliomas, meningiomas, $\mathrm{p}$ & uitary aden & omas) & $71 \%$ & $51 \%$ & $55 \%$ & $59 \%$ & $8 \%$ & $3 \%$ & $60 \%$ & $40 \%$ & $0 \%$ & $0 \%$ \\
\hline Total of studies: & & & 9212 & 20100 & 4639 & 12200 & 414 & 944 & 829 & 255 & 105 & 11 \\
\hline & & & $72 \%$ & $53 \%$ & $50 \%$ & $61 \%$ & $9 \%$ & $8 \%$ & $76 \%$ & $24 \%$ & $13 \%$ & $4 \%$ \\
\hline
\end{tabular}

(1) $95 \%$ CI superior limit $<1$ for OR $<1$, and $95 \% \mathrm{CI}$ inferior limit $>1$ for OR $>1$; (2) ORs decrease together with the increase of exposition and/or latency; n.r. not reported. 
examined the estimated latency is much higher - a minimum of 10-15 years are needed for some of these tumours to develop to detection levels - this is a factor that "dilutes" risk. The percentage of cases or controls exposed for at least 10 years in the above studies is $0 \%[16,18-20]$, less than $5 \%[21,25,27$, $28]$, less than $10 \%[23,24,26,29]$, not given [30]. Hardell in his studies documents a considerably higher percentage of cases and controls (18\% and $13 \%$ respectively) with exposure to MPs of at least $10-15$ years $[1,2]$;

the failure to identify the ipsilateral tumours arising on the side of the head habitually used for calls, mainly in the temporal lobe which is exposed to $97-$ $99 \%$ of the rf radiation emitted during phone use [15]. The radiation decreases rapidly with increasing depth, with consequent further "dilution" of risk due to the detection of tumours in the whole brain mass, for the most part not exposed to radiation. In the 17 Interphone studies, only $2 \%$ of cases with ipsilateral tumours were actually exposed for at least 10 years. Hardell reports $16 \%$ of cases with ipsilateral tumours, some of which involved at least 10-15 years of continuous exposure or latency [1-4];

- the Interphone protocol defines "exposed" subjects having used the phone "at least once a week for at least six months" (which means almost never). Therefore, even if a risk exists, it is "diluted" because of the dominance, in the examined sample, of subjects exposed too little or not at all. The average use of cellphones in subjects considered exposed by Interphone is just 2-5 minutes a day, often for less than 5 years $[5,6]$. These data obviously are barely significant relative to today's intense use of cellphones, especially by those adopting them for work purposes. In Hardell's studies, MP use is reported to be more than 1000 hours for 194 cases and more than 2000 hours for 85 cases over at least 10 years, so that the average use of MPs ranges from over 16 to just more than 32 minutes per day for at least 10 years [1-4];

\section{$\bullet$}

in Interphone, the participation of the identified cases or controls in the study is low: $\leq 50 \%[24,30,31]$, $\leq 60 \%[18,20,28,32,33], \leq 70 \%[16,19,21,23,25]$. In Hardell's studies, participation is always much higher (88-90\%) for both cases and controls [1,2]; owing to the non-blind Interphone protocol, there is often a reduced participation in the study by the nonmobile users initially selected - in particular controls who are not affected by tumours, naturally less interested in the aims of the research than regular users, especially cases affected by tumours - and this represents a further factor of "dilution" of risk estimates. This "control selection bias" is recognised by the Interphone authors themselves [30], but in their view it does not cause reduction in estimated risk by more than $10 \%$, which is true for the overall Interphone data, but in some studies this bias alone can result in a more significant reduction in risk assessment: more than $15 \%[18,28]$, more than $25 \%$ [21-23], and even more than 30\% [20,33]. In Hardell studies, this bias does not occur, as the percentage participation is basically equivalent for the exposed and non-exposed cases and controls [1,2].

Additional factors contributing to "dilution" of risk estimates are:

- the Interphone protocol considers cordless phone users as not exposed, while it is documented that the rf radiation emitted by cordless can even exceed the intensity of a cell phone [34], so much so that Hardell documents s.s. increases in the risk of meningiomas and acoustic nerve neuromas also in people using only cordless [1-4];

- the Interphone study fails to consider other types of malignant and benign head tumours, except gliomas, meningiomas, neuromas and parotid gland tumours. In Hardell's studies increased risks in MP users also involve other types of head tumour, such as low- and high-grade astrocytomas, oligodendrogliomas, other/mixed gliomas, medulloblastomas, ependimomas and other/mixed malignant brain tumours, and also pituitary adenomas and other/mixed benign brain tumours, all of which are considered separately [1-4];

- the risk values of head tumours in three of the Interphone studies [20, 21, 26] even fall off with increased duration of exposure to cellphones and/or latency time, which again seems to content the possibility that cellphone use could provide protection from contracting cancer. In Hardell's studies, the trend for increase in risk as a function of time of MP use or latency is s.s., and the combined use of various types of MPs raises the risk of developing head tumours [1-4].

The combination of these factors in Interphone studies leads to strong underestimation of the risk, and acts such that the majority of risk values (OR) are below 1, often s.s. (Table 1): in the 17 Interphone studies, 76\% of risk values different from 1 are below 1 . The prevalence of OR values below 1 is extremely unusual in most of these studies: $100 \%$ [30], more than $90 \%[22,33]$, more than $80 \%[18,19,24$, $25,32]$, more than $70 \%[20,21,23]$, and the probability of this asymmetric distribution of risk values - which seems to indicate a protective effect - being chance is very low in some of these studies [21, 23, 26, 27, 30, 31], while in other studies $[19,22,24,25,32,33]$, as in the overall data, is practically zero. In Hardell's studies, over $90 \%$ of the risk values are above 1 (with $37 \%$ s.s.), and the probability of this distribution - supporting a carcinogenic effect due to MP use - being due to chance is almost zero.

However, also in the Interphone studies the data obtained using the essential factors for identifying a carcinogenic effect due to cellphone exposure - i.e. significant time use, continuity of use or latency of at least 10 years, and ipsilateral tumour detection - showed a rise often s.s. $>$ $100 \%$ of malignant brain tumour risk $[5,22,24,32]-$ the same being observed for acoustic neuromas [6, 29, 31] and parotid gland tumours $[16,26]$ (Table 2).

Cardis and Sadetzki agreed [7]: "The findings in several studies of an increased risk for glioma among the highest 
Table 2 Increased Risk Values in Some Interphone Studies on the Relationships Between Cellular Phone Use and Head Tumour

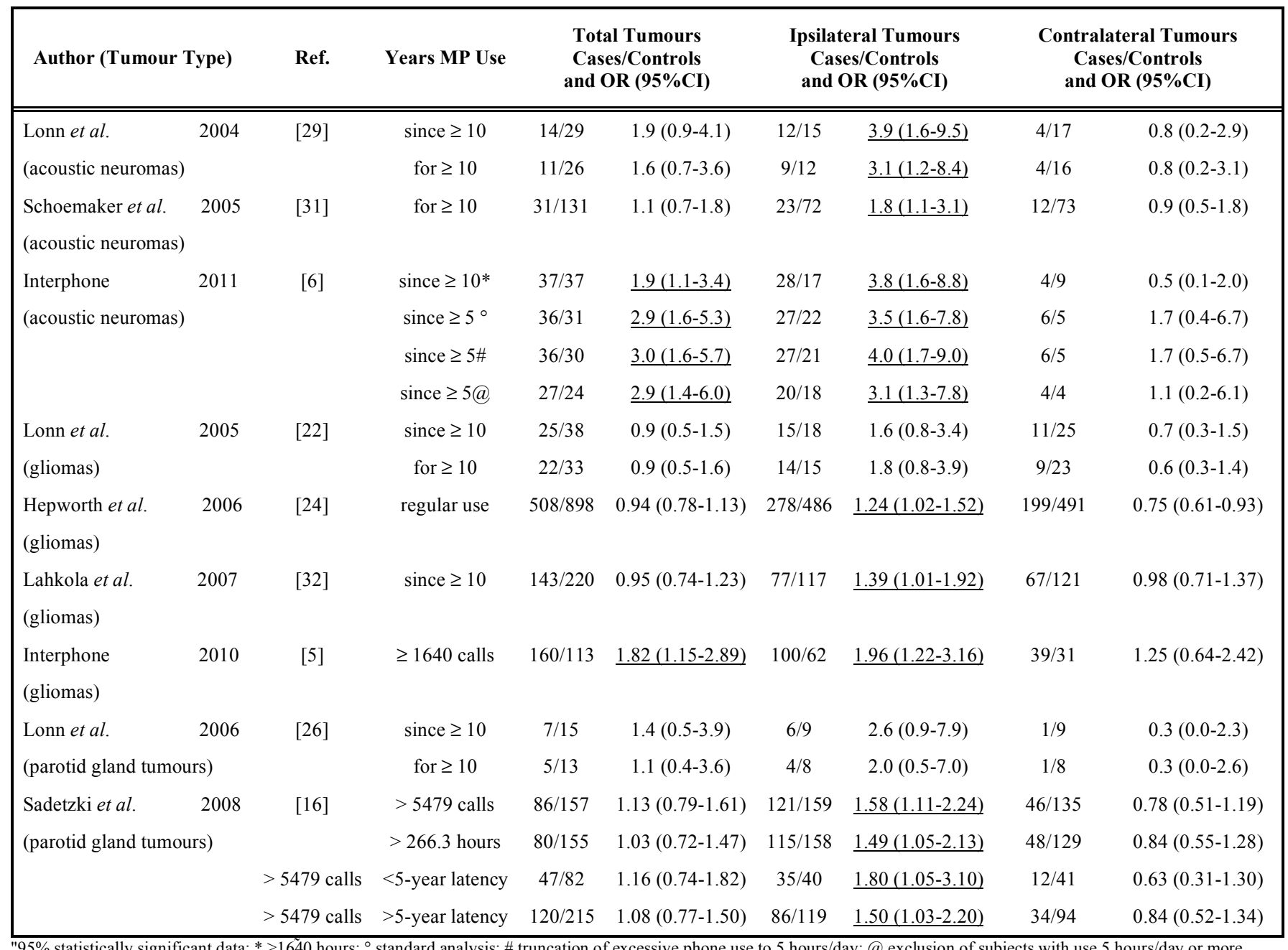

users on the side of the head where the phone was used [1, 5, $22,24,32]-$ and, in Interphone [5], in the temporal lobe are therefore important. These are the findings that would be expected if there was a risk, as these are the a priori relevant exposure variables ... Observations in the highest-exposed group are particularly important, as this could be a sentinel group, signalling effects that might be found in studies with higher and more relevant exposure patterns. Another important issue is that exposure from mobile phones is very localised: most of the rf energy (97-99\% depending on frequency) is absorbed in the brain hemisphere on the side of the head where the phone is used (ipsilateral), mainly in the temporal lobe, and decreases rapidly with increasing depth. The risk, if it exists, is therefore likely to be confined only to a small proportion of the entire brain. Thus, analyses of all brain tumours together are also likely to dilute the risk ... While more studies are needed to confirm or refute these results, indications of an increased risk in high- and longterm users from Interphone and other studies are of concern". They also observed that "Apart from the low use categories, however, the ratios [of ipsi- to contralateral risk values] tended to increase with time since start of use with cumulative call time and with number of calls, suggesting a true effect of mobile-phone use", and concluded stating that "The overall balance of the above-mentioned arguments suggests the existence of a possible association" between cellphone use and increase in brain tumour risk.

Furthermore, the concluding Interphone report on acoustic neuromas [6] - which incorporates the results from the case-control epidemiological studies in all 13 participant countries - corroborates the defects listed above. The data underline how important it is, when evaluating risk, to concentrate on ipsilateral tumours after significant times of daily MP use, and overall times of use and/or latency consistent with development times of the tumours in question. In addition, the fact that increase in acoustic neuroma risk is limited to the side of the head habitually used for cellphone use (ipsilateral with the tumour) gives further confirmation of the existence of a clear cause-effect link (Table 2). However, despite recognising that the negative influence of the "control selection bias" (prevalence of exposed over non-exposed) leads to underestimation of the risk (not more than 15\% according to their estimate), and despite noting the presence of increased risk, s.s. in most cellphone users after adequate latency times, the Interphone authors ambiguously conclude that: "This increase could be due to chance, reporting bias or a causal effect. It is possible too that the interval between introduction of mobile phones and occurrence of the tumour we studied was too short to observe an effect, if there is one, as acoustic neuroma is 
usually a slowly growing tumour". In addition, the previous Interphone data regarding the s.s. increased risk of ipsilateral acoustic neuromas in those using cellphones for at least 10 years [29, 31] (Table 2) are not highlighted, and their weight is cancelled out since they are buried in the overall data of this study. Finally, of Hardell's articles on acoustic neuromas only three are cited; these are among the least significant (not quoted in the present review) and are discarded in a simple comment: "these results are not compatible with our findings", without further explanation.

It should be recalled that the association between MP use and increased head tumour risk was already known and reported in 2007 by the Italian Association of Medical Oncologists - with specific reference to the Hardell metaanalysis [35] - in their monograph "Guidelines for brain tumors" (www.aiom.it), noting a documented "doubling of the risk of brain gliomas and acoustic neuromas among the long term (at least 10 years) users of cellular and cordless phones", and recommending "caution in the use of mobile phones."

In fact, the meta-analyses of Hardell [35, 36], Khurana [37], Kundi [38], and ourselves [39], including the literature data on head tumours in people with MP latency or continuous use of at least 10 years - and thus, besides Hardell's data [1-4, 36], also part of the Interphone data [23$25,29,31,32]$ - show large and s.s. increases $(100 \%)$ of the risk of ipsilateral gliomas with high level of malignancy, and sizable and s.s. increases (50-140\%) of the risk of ipsilateral acoustic neuromas (Table 3). These increases are smaller than those found by Hardell in the pooled analyses of his data alone $[1,2,4]$, being "diluted" with the Interphone data corresponding to the requirements indicated above. Indeed, by splitting the overall risk data of these meta-analyses according to their source, only Hardell's data are systematically above 1 (90-100\%), of which $50-90 \%$ are s.s., whereas Interphone data include $50-70 \%$ of risk values below 1, a proportion of which (up to over 20\%) are s.s. Moreover, when only ipsilateral data are considered [37], even $100 \%$ of the Interphone risk values are above 1 , of which $29 \%$ are s.s.

The reasons underlying the discrepancy between Hardell's positive data and the negative Interphone findings become clear by close examination of one of the latest articles from Hardell [40], who carried out a new metaanalysis, taking into account the Interphone data as well as his own: while the data overall do not show any increase in head tumour risk in MP users, limiting the meta-analysis to just ipsilateral tumours in individuals with at least 10-year latency, a s.s. doubling in risk is found for gliomas and a $60 \%$ increase in risk is found for acoustic neuromas. Furthermore, while in Hardell's data more than $90 \%$ of risk values are above 1 , for the main part (more than 50\%) s.s., this is the case for the Interphone data only when the analysis is limited to ipsilateral tumours in individuals with

Table 3. Results of the Meta-Analyses of Case-Control Studies on the Head Tumour Risk in MP Users (Cases/Controls):OR; 95\%CI

\begin{tabular}{|c|c|c|c|c|c|c|c|c|}
\hline Author & . & Ref & \multicolumn{2}{|c|}{ All } & \multicolumn{2}{|c|}{ Ipsilateral } & \multicolumn{2}{|c|}{ Contralateral } \\
\hline \multicolumn{9}{|l|}{ gliomas } \\
\hline Hardell & '07/'08 & {$[35,36]^{*}$} & (338/511): & $1.2 ; 0.8-1.9$ & (n.s.): & $2.0 ; 1.2-3.4$ & (n.s.): & $1.1 ; 0.6-2.0$ \\
\hline Kundi & '09 & {$[38]^{*}$} & (233/330): & $\underline{1.5 ; 1.2-1.8}$ & (n.s.): & $\underline{1.9 ; 1.4-2.4}$ & \multicolumn{2}{|c|}{ (n.a.) } \\
\hline Khurana & '09 & {$[37]^{*}$} & (233/330): & $\underline{1.3 ; 1.1-1.6}$ & (118/145): & $\underline{1.9 ; 1.4-2.4}$ & (93/150): & $1.2 ; 0.9-1.7$ \\
\hline Levis & '11 & {$[39]^{*}$} & (240/297): & $1.0 ; 0.8-1.2$ & (259/371): & $\underline{1.6 ; 1.2-2.0}$ & $(154 / 263):$ & $1.0 ; 0.7-1.2$ \\
\hline Myung $^{\circ}$ & '09 & {$[55]^{*}$} & (n.s.): & $\underline{1.5 ; 1.3-1.9}$ & \multicolumn{2}{|c|}{ (n.a.) } & \multicolumn{2}{|c|}{ (n.a.) } \\
\hline Myung ${ }^{\#}$ & '09 & {$[55]$} & (n.s.): & $1.0 ; 0.8-1.2$ & \multicolumn{2}{|c|}{ (n.a.) } & \multicolumn{2}{|c|}{ (n.a.) } \\
\hline Lahkola & '06 & {$[42]$} & (339/n.s.): & $1.0 ; 0.8-1.2$ & \multicolumn{2}{|c|}{ (n.a.) } & \multicolumn{2}{|c|}{ (n.a.) } \\
\hline Ahlbom & '09 & [41] & (n.s.): & $1.1 ; 0.8-1.4$ & \multicolumn{2}{|c|}{ (n.a.) } & \multicolumn{2}{|c|}{ (n.a.) } \\
\hline Repacholi & '11 & {$[58]$} & (n.s.): & $1.4 ; 0.8-2.3$ & \multicolumn{2}{|c|}{ (n.a.) } & \multicolumn{2}{|c|}{ (n.a.) } \\
\hline \multicolumn{9}{|l|}{ neuromas } \\
\hline Hardell & '07/'08 & {$[35,36]^{*}$} & $(83 / 355):$ & $1.3 ; 0.6-2.8$ & (53/167): & $\underline{2.4 ; 1.1-5.3}$ & $(30 / 151):$ & $1.2 ; 0.7-2.2$ \\
\hline Kundi & '09 & {$[38]^{*}$} & $(67 / 311):$ & $1.3 ; 1.0-1.9$ & (n.s.): & $\underline{1.5 ; 1.1-2.5}$ & \multicolumn{2}{|c|}{ (n.a.) } \\
\hline Khurana & '09 & {$[37]^{*}$} & $(67 / 311):$ & $1.3 ; 1.0-1.9$ & (41/152): & $\underline{1.6 ; 1.1-2.4}$ & (26/134): & $1.2 ; 0.4-1.0$ \\
\hline Levis & '11 & {$[39]^{*}$} & (98/296): & $1.2 ; 0.9-1.7$ & (125/504): & $\underline{1.7 ; 1.2-2.6}$ & $(46 / 240):$ & $1.2 ; 0.9-1.7$ \\
\hline Myung $^{\circ}$ & '09 & {$[55]^{*}$} & (n.s.): & $\underline{1.7 ; 1.3-2.1}$ & \multicolumn{2}{|c|}{ (n.a.) } & \multicolumn{2}{|c|}{ (n.a.) } \\
\hline Myung ${ }^{\#}$ & '09 & {$[55]$} & (n.s.): & $1.0 ; 0.8-1.3$ & \multicolumn{2}{|c|}{ (n.a.) } & \multicolumn{2}{|c|}{ (n.a.) } \\
\hline Lahkola & '06 & [42] & (167/n.s.): & $1.1 ; 0.9-1.3$ & \multicolumn{2}{|c|}{ (n.a.) } & \multicolumn{2}{|c|}{ (n.a.) } \\
\hline Ahlbom & '09 & {$[41]$} & (n.s.): & $1.4 ; 0.7-2.5$ & \multicolumn{2}{|c|}{ (n.a.) } & \multicolumn{2}{|c|}{ (n.a.) } \\
\hline Repacholi & '11 & {$[58]$} & (n.s.): & $1.4 ; 0.7-2.5$ & \multicolumn{2}{|c|}{ (n.a.) } & \multicolumn{2}{|c|}{ (n.a.) } \\
\hline
\end{tabular}

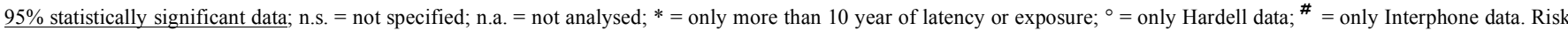
values for meningiomas are all not significant (not shown). 
at least 10 -year latency: $90 \%$ of risk values are above $1-$ of which $22 \%$ are s.s.

In contrast to the above meta-analyses, that of Ahlbom/ICNIRP [41] is based on data of the first (19992003) case-control studies including those of Hardell (not quoted in the present review), in other words without cases with $\geq 5-10$ year latency. Moreover, it reports selectively chosen data from Hardell's 2006 papers, including his pooled-analyses $[1,2]$ referred to subjects with $\geq 5$ year latency but does not highlight, for those with $\geq 10$ year latency, the much more significant data on increases of ipsilateral head tumour risk, although these are clearly indicated in Hardell's papers. Besides, it does not consider the recent studies by Hardell [3, 35-37] or those of Interphone $[22,24,29,31,32]$ which report s.s. increases of ipsilateral head tumour risk after $\geq 10$-year latency or use of cellular phones (Table 2). Consequently, the Ahlbom metaanalysis gives results that lack any indication of head tumour risk from MP use or latency up to $\geq 10$ years (Table 3 ), emphasising the complete incompatibility between the two data sources: $83 \%$ of Hardell's risk data (OR) are $>1$, of which $43 \%$ are s.s., while the Interphone data are largely $(73 \%)<1$, of which $11 \%$ are s.s.

The same pattern of "negative" results is shown by the meta-analysis performed by five national Interphone groups [42], based on a "targeted" choice of cases with $<5-10$ year latency, from the first (1999-2002) US and Hardell's studies and even from a cohort study (not quoted in the present review), as well as from a few Interphone studies [22, 23, 31] (Table 3).

In a commentary [43] with a very telling title "Observational studies may conceal a weakly elevated risk under the appearance of consistently reduced risk", Saracci and Pearce criticised another Lahkola meta-analysis on meningioma risk in mobile phone users in 5 North European countries [33]: "A key finding is that risk is decreased in all five countries: $\mathrm{OR}=0.87$ not significant (n.s.) for Denmark, 0.75 s.s. for Finland, 0.85 n.s. for Norway, 0.68 s.s. for Sweden and 0.72 s.s. for Southeast England. Can these findings be due to chance? On the null hypothesis of no effect of mobile phone use, the probability of a country specific OR being below 1.0 is $50 \%$ and the probability of all five OR being below 1.0 by chance is only $3 \%$. Also one would expect zero or one OR to be statistically significant at $\mathrm{P}<0.10$ whereas three are observed. In addition, the already noted systematic pattern of decreased risk in the aggregate data indicates that these findings are unlikely to be due to chance. If chance is an implausible explanation, consideration must shift to uncontrolled confounding and/or biasing factors". The same picture is given by Lahkola metaanalyses on glioma [32] and total brain tumour risks [30] (Table 4). Overall, in the three Lahkola studies all the 22 ORs are $<1$, of which 12 are s.s., and the probability of this being by chance is almost zero. All the more, such a systematically reduced tumour risk must be attributed to uncontrolled confounding and/or biasing factors.

In May 2011, with a delay of six years over the date initially announced, IARC convened a working group to assess the carcinogenic risks associated with $\mathrm{rf}$ radiation, including that from MPs. The final report [44], published ahead of a related monograph (due around Spring 2012), dedicates a considerable part to analysis of studies on head tumour risk due to MP use, and states that: "The Interphone study [5], a multicentre case-control study, is the largest investigation so far of mobile phone use and brain tumours, including glioma, acoustic neuroma, and meningioma ... There was suggestion of an increased risk for ipsilateral exposure (on the same side of the head as the tumour) and for tumours in the temporal lobe, where rf exposure is highest ... The OR for glioma increased with increasing $\mathrm{rf}$ dose for exposures 7 years or more before diagnosis, whereas there was no association with estimated dose for exposures less than 7 years before diagnosis ... A Swedish research group did a pooled analysis of two very similar studies of associations between mobile and cordless phone use and glioma, acoustic neuroma, and meningioma (see our ref. 4) ... Participants who had used a mobile phone for more than 1 year had an OR for glioma of 1.3 s.s. The OR increased with increasing time since first use and with total call time, reaching 3.2 s.s. for more than $2000 \mathrm{~h}$ of use. Ipsilateral use of the mobile phone was associated with higher risk. Similar findings were reported for use of cordless phones (see our ref. 4). Although both the Interphone study and the Swedish pooled-analysis are susceptible to bias - due to recall error and selection for participation - the Working Group concluded that the findings could not be dismissed as reflecting bias alone, and that a causal interpretation between mobile phone $\mathrm{rf}$ exposure and glioma is possible. A similar conclusion was drawn from these two studies for acoustic neuroma, although the case numbers were substantially smaller than for glioma. Additionally, a study from Japan (see our ref. [45]) found some evidence of an increased risk for acoustic neuroma associated with ipsilateral mobile phone use".

The IARC/OMS conclusions [44] are drawn by Samet (overall Chairman of the Working Group) and by Wild (the IARC Director). Samet stated: "The evidence, while still accumulating, is strong enough to support a conclusion and the 2B classification (corresponding to the IARC classification as "possibly carcinogenic to humans"). The conclusion means that there could be some risk, and therefore we need to keep a close watch for a link between cell phone and cancer risk". Whilst Wild noted: "Given the potential consequences for public health of this classification and findings, it is important that additional research be conducted into the long-term heavy use of mobile phones. Pending the availability of such information, it is important to take pragmatic measures to reduce exposure such as hands-free devices or texting".

Saracci, who participated in the IARC working group on rf assessment, emphasised his criticisms of the Interphone studies [5,6] in an editorial [46], where he explicitly states that: "The preparation of the overall final reports has been long and laborious, with results difficult to interpret. This fact immediately jumps to the attention once it is seen that the relative risk (odds ratio) for cerebral gliomas is 0.70 s.s. comparing cellphone users and non-users. A reduction in risk is found in 10 of the 13 participant countries, which makes it being due to chance unlikely $(\mathrm{p}=\sim 0.05)$. This leaves two alternative interpretations: either there is present a causal protective effect of cellphones, or a biasing of the results has arisen during the study.... Since the study in question is simply observational, the logical explanation is 
Table 4 Risk Values in Three Lahkola Studies on the Relationships Between Cellular Phone Use and Head Tumours

\begin{tabular}{|c|c|c|c|c|c|c|}
\hline Author & Year & Tumour Type & Ref. & Cases/Controls): & $(\mathrm{OR} ; 95 \% \mathrm{CI}$ & Notes \\
\hline \multirow[t]{7}{*}{ Lahkola } & 2005 & brain tumours & {$[30]$} & (726/777): & $0.55 ; 0.39-0.77 *$ & regular use, full participants \\
\hline & & & & $(103 / 321)$ & $0.62 ; 0.26-1.51$ & regular use, incomplete participants \\
\hline & & & & (829/1098): & $0.73 ; 0.56-0.96^{*}$ & regular use, all participants \\
\hline & & & & $(245 / 287):$ & $0.52 ; 0.28-0.97 *$ & regular use, females, full participants \\
\hline & & & & (271/422): & $0.94 ; 0.62-1.42$ & regular use, females, total participants \\
\hline & & & & (309/359): & $0.56 ; 0.38-0.84 *$ & regular use, males, full participants \\
\hline & & & & (348/454): & $0.61 ; 0.43-0.88^{*}$ & regular use, males, total participants \\
\hline \multirow[t]{7}{*}{ Lahkola } & 2007 & gliomas & {$[32]$} & (1521/3301): & $0.78 ; 0.68-0.91 *$ & all: regular use \\
\hline & & & & (1389/2945): & $0.63 ; 0.48-0.82 *$ & all: "ever use" \\
\hline & & & & (247/819): & $0.70 ; 0.51-0.96^{*}$ & only Denmark \\
\hline & & & & (266/870): & $0.80 ; 0.61-1.09$ & only Finland \\
\hline & & & & (284/353): & $0.62 ; 0.42-0.91 *$ & only Norway \\
\hline & & & & (363/629): & $0.82 ; 0.61-1.09$ & only Sweden \\
\hline & & & & $(361 / 630):$ & $0.95 ; 0.70-1.29$ & only Southeast England \\
\hline \multirow[t]{6}{*}{ Lahkola } & 2008 & meningiomas & [33] & (1209/3299): & $0.76 ; 0.65-0.89^{*}$ & total subjects \\
\hline & & & & (173/819): & $0.87 ; 0.60-1.27$ & only Denmark \\
\hline & & & & (334/870): & $0.75 ; 0.56-1.01$ & only Finland \\
\hline & & & & (206/353): & $0.85 ; 0.57-1.29$ & only Norway \\
\hline & & & & (271/627): & $0.68 ; 0.49-0.94 *$ & only Sweden \\
\hline & & & & (225/630): & $0.72 ; 0.51-1.01$ & only Southeast England \\
\hline
\end{tabular}

that there is a bias, due to one or more factors that need to be identified (if possible) .... A bias that reduces the risk implies that any increase in observed risk is actually underestimated: this is particularly noteworthy if found where a priori most probable, that is, for highest levels of exposure". Continuing, Saracci observed that: "In both the Interphone study and the Swedish (Hardell: 1-4) studies, the results for gliomas are not found for meningiomas, tumours that are slower to develop. This argues against the presence of some generalised biasing factor, but for the reliability of the results obtained for gliomas".

\section{DISCUSSION}

According to Hardell and his co-workers [47-52] there are many biases and flaws in the non-blind Interphone protocol that lie behind the large prevalence of risk values below 1 in the overall results, giving rise to a systematic underestimate of the risk (Box 1). On the other hand, the double-blind protocol by Hardell producing positive results is without apparent errors or conditioning (Box 2), the results indicating a cause-effect relationship supported by biological plausibility [44, 53, 54].

We have already published a more detailed comparison of Hardell's and Interphone protocols and results [39]. Moreover, the results of our meta-analyses confirm that to determine true risk of head tumours due to MP radiation it is vital to identify the laterality of head tumour localisation relative to the habitual laterality of MP use, in subjects with at least 10 year use or latency of cellular and cordless phones.

The discrepancy between the positive data of Hardell and the negative data from Interphone is also highlighted by Myung et al., [55] who performed a random-effect model meta-analysis of 24 case-control studies. These authors observed an s.s. positive association between MP use and increased head cancer risk in ten studies using blinding ("high-quality studies", including seven studies by Hardell, only one by Interphone, and two by other groups), whereas a negative association (i.e. a reduced risk, suggesting a protective effect) was observed in fourteen studies not using blinding ("low-quality studies", including twelve by Interphone, two by other groups, any by Hardell). Elements in the method used to evaluate the "quality" of the studies were:

- $\quad$ blind or non-blind protocol;

- $\quad$ presence or absence of participation and selection biases of cases and controls;

- $\quad$ relevant or marginal MP exposure;

- $\quad$ adequate or inadequate latency or overall time of MP use;

- $\quad$ scrutiny of tumour laterality;

- funding by independent sources or by cellphone companies. 
Box 1. Methodological Errors and Bias of Negative Interphone Studies, Based on a Non-Blind Protocol, on Tumour Risk from Cellular Phone Use

1 Inadequate assessment of the "regular use of cell phones", defined as "at least 1 phone call per week, for at least 6 months", giving rise to an average use of 2-5 min/day.

2 Inadequate latency time in "exposed" subjects in relation to the time required for diagnosing the tumours concerned: less than $10 \%$ of cases in 17 Interphone studies have latency time of at least 10 years.

3 Failure to include cordless users, even though they are exposed: they are included in the non-exposed group.

4 Failure to include the under-20 in 3 studies, although this group has high exposure.

5 Failure to include residents of rural areas (except 1 study), although this group has high exposure.

6 Failure to include subjects who had died or were too weak to respond to the interview carried out during post-operatory convalescence.

7 Failure to identify tumour laterality in relation to laterality of MP use: only $2 \%$ of cases with ipsilateral tumours are exposed for $\geq 10$ years. The possible increase in ipsilateral tumours is very much reduced by the missing increase in total and especially contralateral tumours.

8 Failure to consider other types of malignant and benign head tumour, except gliomas, neuromas, meningiomas, and parotid gland tumours.

9, 10 Participation and selection bias: since a non-blind protocol is used, the participation of cases and controls is reduced to $60 \%$, at times $<40 \%$, with prevalence of the exposed compared to non-exposed controls.

11 Delayed interviews: the controls are interviewed at a later stage than the cases (up to $>9$ months). For this reason, given the rapid spread of MPs, the control group contains more exposed subjects than the case group.

12 Data collection bias: as it is impossible to collect responses from hospitalized cases that are frail, the information is collected from a relative (up to $40 \%$ of cases) with consequent data uncertainty.

13 Type and time for the interviews. Cases: bedside face-to-face by nurses or medical students during hospitalisation. Controls: face-to-face, usually at their homes.

14 Attribution bias in laterality of MP use: the patient, often still in a confused state during the post-operatory period, may report the most recent laterality of use which, owing to the disturbances brought about by the tumour, may not actually be the side habitually used before the development of the tumour.

15 Assessment of exposure: computer-guided personal interviews, with knowledge of whether the subject was a case or a control, exposed or not exposed.

16 Data processing: not stated (not blind ?).

17 Documentation bias: in the bibliography cited to support the Interphone findings as reassuring, negative studies are widely reported and discussed, without critical assessment of the experimental protocol and results. Instead, the positive studies of Hardell group are regularly ignored, underevaluated, or even selectively chosen.

18 Funding bias: the findings from Interphone studies, which are co-funded by the international and national cellphone companies, are publicised as being fully reassuring - even though at times they include positive data indicative of increased carcinogenic risk (e.g. for ipsilateral tumours or in the subgroup exposed for $\geq 10$ years, or in rural residents).

Box 2. Reliability of Positive Hardell Studies, Based on a Double-Blind Protocol, on Tumour Risk from Cellular and Cordless Phone Use

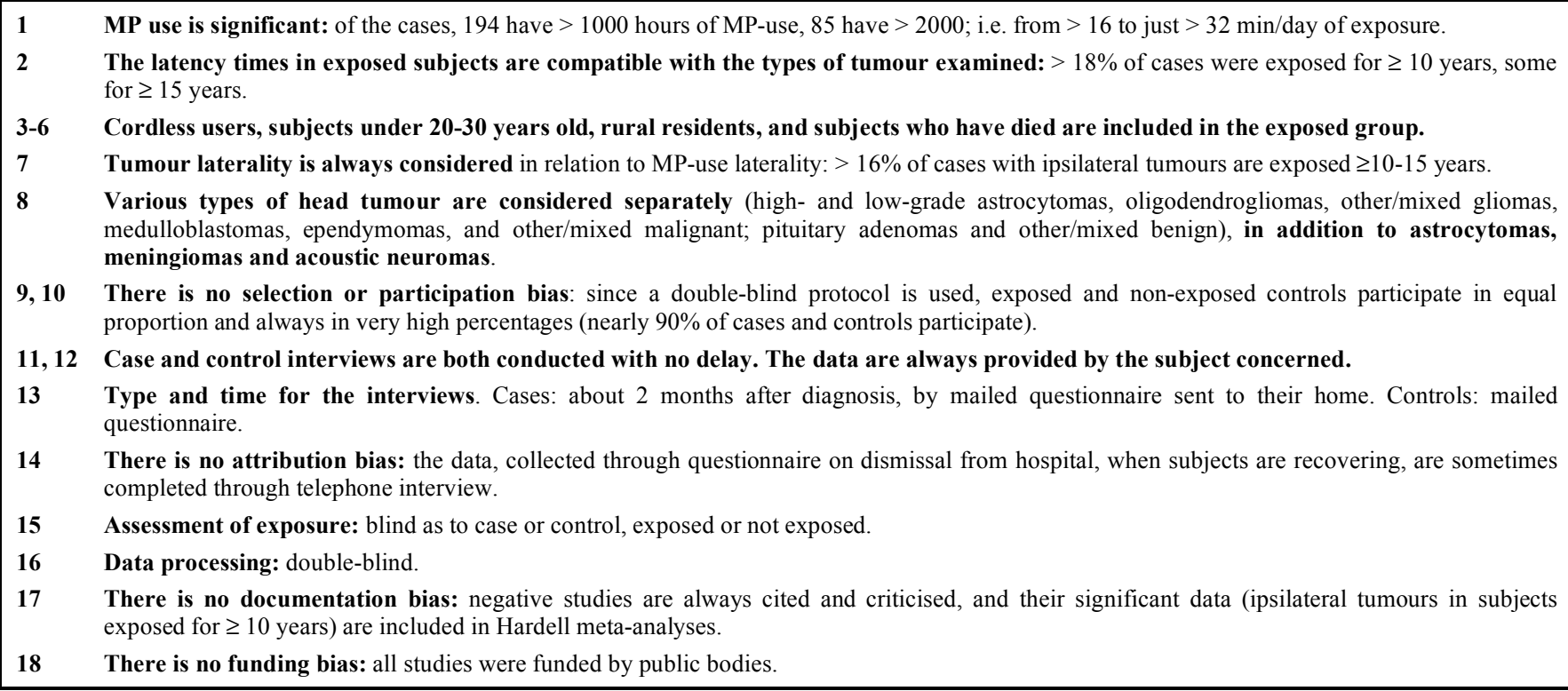

These authors reached the following conclusion: "We feel the need to mention the funding sources for each research group because it is possible that these may have influenced the respective study designs and results".
The Hardell's group was only ever supported by grants from public bodies, whereas the Interphone-related studies received funding through the Quality of Life and Management of Living Resources programme of the 
European Union and the International Union Against Cancer, but in turn the latter received funding for the Interphone studies from the Mobile Manufacturers Forum and the Global System for Mobile Communication Association [56]. In addition to the above private funds, several authors participating in the Interphone study received additional funding from their national MP companies [5, 6, 24, 25, 27, 30-33] or from other private companies [23], such that a substantial portion of the Interphone Study funding came from the cellphone industry. These additional funds are not specified in the Interphone protocol [56], and the agreements regulating access to the experimental results and the control of their use by the array of national cellphone and other private companies involved are not known.

Nevertheless, of the authors of the above "negative" studies, ten do not make any declaration about conflict of interest [6, 21-24, 29-33], three state "conflict of interest: none declared" (it is not clear whether this came from the authors or from the editor) $[20,25,26]$, while four declare "conflict of interest: none" [18, 19, 27, 28].

We are therefore in full agreement with the conclusion of Cardis and Sadetzki [15] that "While more studies are needed to confirm or refute these results, indications of an increased risk in high- and long-term users from Interphone and other studies are of concern ... There are now more than 4 billion people, including children, using mobile phones. Even a small risk at the individual level could eventually result in a considerable number of tumours and become an important public-health issue. Simple and low-cost measures, such as the use of text messages, hands-free kits and/or the loud-speaker mode of the phone could substantially reduce exposure to the brain from mobile phones. Therefore, until definitive scientific answers are available, the adoption of such precautions, particularly among young people, is advisable".

These measures - simple and inexpensive for those who put them into practice, although less profitable for the cellphone companies - are set out on various web sites (www.sante radiofrequences.org; www.devradavis.com; www.truth-out.org/article/twenty-appeal-against-cell-phone).

Despite all the evidence above, most of the Interphone authors (four of whom participated in the IARC assessment group [44]) are now launching the "Cosmos" epidemiology study [57] on MP users in five European countries "who will be followed up for 25+ years". This new study does not involve Cardis and Sadetzki and is again co-funded by the cellphone companies. The "Cosmos" authors continue to support "the current exposure guidelines developed by ICNIRP to minimize the effect of tissue heating from exposure to $\mathrm{rf}$ - and thus also to mobile phone rf emissions which to date is the only known biological effect." Moreover, they state: "In a recent risk assessment by the European Commission it was concluded from three independent lines of evidence (epidemiological, animal, and in vitro studies) that exposure to rf below these exposure guidelines is unlikely to lead to an increase in cancer", and that: "no seemingly plausible biophysical or biological mechanism has been identified in relation to potential health effects of rf'".
Furthermore, the authors of the recent review by Interphone/ICNIRP [58] - three of whom were invited to participate in the IARC evaluation group [44] - maintain that: "the results of the principal epidemiology studies, the Interphone Study, and the studies by Hardell et al. are inconsistent", and that: "there are also insufficient data to make any determinants about risks for children and longterm use ( $\geq 10$ years) by adults".

\section{CONCLUSION}

While recognizing that mobile telephony is an outstanding technology of inestimable value, responsible science must raise awareness of the already documented risks.

The unfolding story of the carcinogenic action of the frequent and long-term use of mobile phones looks set to become another case of history repeating itself - following in the tracks of ionizing radiation, asbestos, tobacco smoke, and many other now demonstrated human carcinogens where evidence of harm was officially recognised only a score or even more years after the initial warnings. In view of the evidence already accumulated, now we can act early, so future generations will not be left once again to regret our inaction. It is our duty and responsibility as scientists.

Summing up, we believe that today there is already sufficient epidemiological evidence to warrant application of the Precautionary Principle aimed at:

- $\quad$ setting limits for MP rf emissions that are really precautionary;

- $\quad$ providing accurate information about the risks from exposure to MPs, with low-cost voluntary options ("prudent avoidance") based on caution in the use of MPs;

- $\quad$ awareness-raising in schools through a campaign on the use of wireless technologies;

- $\quad$ discouraging the use of MPs by minors.

\section{ACKNOWLEDGEMENTS}

Financial support from University of Padova - Italy. The authors wish to thank Dr Susan Biggin for valuable suggestions and language/editorial input in preparation of the manuscript.

\section{AUTHORS' CONTRIBUTIONS}

A.G.L. and S.G. are responsible for the collection and analysis of data, and for writing the manuscript. N.M. performed the meta-analyses. All the authors contributed to the discussion of data and draw conclusions, and approved the final manuscript.

\section{CONFLICT OF INTERESTS:}

The authors declare that they have no conflicting interests.

\section{ABBREVIATIONS}

$\mathrm{CI}=$ Confidence Interval

IARC $=$ International Agency for Research on Cancer 


$$
\begin{array}{ll}
\text { ICNIRP } & \text { International Commission for Non-Ionizing } \\
& \text { Radiation Protection } \\
\text { MP } & \text { Mobile Phone } \\
\text { n.s. } & =\text { statistically not significant } \\
\text { rf } & =\text { radiofrequency } \\
\text { OR } & =\text { Odds Ratio } \\
\text { S.s. } & =95 \% \text { statistically significant. }
\end{array}
$$

\section{REFERENCES}

[1] Hardell L, Carlberg M, Hansson-Mild K. Pooled analysis of two case-control studies on use of cellular and cordless telephones and the risk for malignant brain tumours diagnosed in 1997-2003. Int Arch Occup Environ Health 2006; 79: 630-9.

[2] Hardell L, Carlberg M, Hansson-Mild K. Pooled analysis of two case-control studies on the use of cellular and cordless telephones and the risk of benign brain tumours diagnosed during 1997-2003. Int J Oncol 2006; 28: 509-18.

[3] Hardell L, Carlberg M. Mobile phones, cordless phones and the risk for brain tumours. Int J Oncol 2009; 35: 5-17.

[4] Hardell L, Carlberg M, Hansson MK. Pooled analyses of casecontrol studies on malignant brain tumours and the use of mobile and cordless phones including living and deceased subjects. Int $\mathbf{J}$ Oncol 2011; 38: 1465-74

[5] The Interphone Study Group. Brain tumour risk in relation to mobile telephone use: results of the INTERPHONE international case-control study. Int J Epidemiol 2010; 39: 675-94.

[6] The Interphone Study Group. Acoustic neuroma risk in relation to mobile telephone use: results of the INTERPHONE international case-control study. Cancer Epidemiol 2011; 35: 453-64

[7] International Agency for Research on Cancer (IARC). Interphone study reports on mobile phone use and brain cancer risk 2010; press release no. 200.17.05.2010. Available from: www.iarc.fr/en/ media-centre/pr/2010/pdfs/pr200.E.pdf

[8] World Health Organization (WHO). No evidence linking cell phone use to risk of brain tumors. Available from: www.who. int/mediacentre/factsheets/fs 193/en/index.html

[9] International Commission on Non-Ionizing Radiation Protection (ICNIRP). Note on the Interphone Publication 2010. Available from: www.icnirp.org

[10] International Union Against Cancer (UICC). Interphone study reports on mobile phone use and brain cancer risk 2010. Available from: www.uicc.org/print/7639

[11] Food and Drug Administration (FDA). No evidence linking cellphone use to risk of brain tumors. FDA Consumer Health Information 2010. Available from: www.fda.gov/ForConsumers/ ConsumerUpdates/ucm212273.htm

[12] National Cancer Institute (NCI). Study finds no overall increased brain tumor risk from cell phones. NCI Cancer Bulletin 2010. Available from: www.cancer.gov/ncicancerbulletin/051810/page10

[13] Health Protection Agency (HPA). The interphone study has not established an increase in brain cancer 2010. Available from: www.hpa.org.uk/HPA/Topics/Radiation/UnderstandingRadiation/1 158934607786

[14] Saracci R, Samet J. Commentary: call me on my mobile phone or better not? a look at the INTERPHONE study results. Int J Epidemiol 2010; 39: 695-8.

[15] Cardis E, Sadetzki S. Indications of possible brain-tumour risk in mobile-phone studies: should we be concerned? Occup Environ Med 2011; 68: 169-71.

[16] Sadetzki S, Chetrit A, Jarus-Hakak A, et al. Cellular phone use and risk of benign and malignant parotid gland tumors: a nationwide case-control study. Am J Epidemiol 2008; 167: 457-67.

[17] Havas M. Interphone Study: it's not just brain tumors. Available from: www.magdahavas.com/2010/05/17/interphone_parotid_gland_tumors

[18] Takebayashi T, Akiba S, Kikuchi Y, et al. Mobile phone use and acoustic neuroma risk in Japan. Occup Environ Med 2006; 63: 8027.

[19] Klaeboe L, Blaasaas KG, Tynes T. Use of mobile phones in Norway and risk of intracranial tumours. Eur J Cancer Prev 2007; 16: $158-64$.
[20] Schlehofer B, Schlaefer K, Blettner M, et al. Environmental risk factors for sporadic acoustic neuroma (Interphone Study Group, Germany). Eur J Cancer 2007; 43: 1741-7.

[21] Christensen HC, Schuz J, Kosteljanetz M, et al. Cellular telephone use and risk of acoustic neuroma. Am J Epidemiol 2004; 159: 27783 .

[22] Lonn S, Ahlbom A, Hall P, et al. Swedish Interphone study group. Long-term mobile phone use and brain tumor risk. Am J Epidemiol 2005; 161: 526-35.

[23] Christensen HC, Schuz J, Kosteljanetz M, et al. Cellular telephones and risk for brain tumors: a population-based, incident case-control study. Neurology 2005; 64: 1189-95.

[24] Hepworth SJ, Schoemaker MJ, Muir KR, et al. Mobile phone use and risk of glioma in adults: case-control study. Brit Med J 2006; 332: 883-7.

[25] Schuz J, Bohler E, Berg G, et al. Cellular phones, cordless phones, and the risks of glioma and meningioma (Interphone study group, Germany). Am J Epidemiol 2006; 163: 512-20.

[26] Lonn S, Ahlbom A, Christensen HC, et al. Mobile phone use and risk of parotid gland tumor. Am J Epidemiol 2006; 164: 637-43.

[27] Hours M, Bernard M, Montestrucq L, et al. Cell phones and risk of brain and acoustic nerve tumours: the French Interphone casecontrol study. Rev Epidemiol Sante Publique 2007; 55: 321-32.

[28] Takebayashi T, Varsier N, Kikuchi Y, et al. Mobile phone use, exposure to radiofrequency electromagnetic field, and brain tumour: a case-control study. Br J Cancer 2008; 98: 652-9.

[29] Lonn S, Ahlbom A, Hall P, et al. Mobile phone use and the risk of acoustic neuroma. Epidemiology 2004; 15: 653-9.

[30] Lahkola A, Salminen T, Auvinen A. Selection bias due to differential participation in a case-control study of mobile phone use and brain tumors. Ann Epidemiol 2005; 15: 321-5.

[31] Schoemaker MJ, Swerdlow AJ, Ahlbom A, et al. Mobile phone use and risk of acoustic neuroma: results of the Interphone case-control study in five North European countries. Br J Cancer 2005; 93: 8428.

[32] Lahkola A, Auvinen A, Raitanen J, et al. Mobile phone use and risk of glioma in 5 North European countries. Int J Cancer 2007; 120: $1769-75$.

[33] Lahkola A, Salminen T, Raitanen J, et al. Meningioma and mobile phone use: a collaborative case-control study in five North European countries. Int J Epidemiol 2008; 37: 1304-13.

[34] Redmayne M, Inyang I, Dimitriaidis C, et al. Cordless telephone use: implications for mobile phone research. J Environ Monit 2010; 12: 809-12.

[35] Hardell L, Carlberg M, Soderqvist F, et al. Long-term use of cellular phones and brain tumours: Increased risk associated with use for $>$ or $=10$ years. Occup Environ Med 2007; 64: 626-32.

[36] Hardell L, Carlberg M, Soderqvist F, et al. Meta-analysis of longterm mobile phone use and the association with brain tumours. Int $\mathrm{J}$ Oncol 2008; 32: 1097-103.

[37] Khurana VG, Teo C, Kundi M, et al. Cell phones and brain tumors: a review including the long-term epidemiologic data. Surg Neurol 2009; 72: 205-14.

[38] Kundi M. The controversy about a possible relationship between mobile phone use and cancer. Environ Health Perspect 2009; 117: 316-24.

[39] Levis AG, Minicuci N, Ricci P, et al. Mobile phones and head tumours. The discrepancies in cause-effect relationships in the epidemiological studies - how do they arise? Environ Health 2011; 10: 59. Available from: http://www.ehjournal.net/content/10/1/59

[40] Hardell L, Carlberg M, Hansson-Mild K. Epidemiological evidence for an association between use of wireless phones and tumour diseases. Pathophysiology 2009; 16: 113-22.

[41] Ahlbom A, Feychting M, Green A, et al. Epidemiologic evidence on mobile phones and tumour risk: a review. Epidemiol 2009; 20: 639-52.

[42] Lahkola A, Tokola K, Auvinen A. Meta-analysis of mobile phone use and intracranial tumors. Scand J Work Environ Health 2006; 32: 171-7.

[43] Saracci R, Pearce N. Commentary: observational studies may conceal a weakly elevated risk under the appearance of consistently reduced risk. Int J Epidemiol 2008; 37: 1313-5.

[44] International Agency for Research on Cancer (IARC). Carcinogenicity of radiofrequency electromagnetic fields. The Lancet-Oncology 2011; 12: 624-6 (press release n. 208, 31 May 
2011: IARC classifies radiofrequency electromagnetic fields as possibly carcinogenic to humans).

[45] Sato Y, Akiba S, Kubo O, Yamaguchi N. A case-case study of mobile phone use and acoustic neuroma risk in Japan. Bioelectromagnetics 2011; 32: 85-93.

[46] Saracci R. Electromagnetic fields from wireless phones declared "possibly carcinogenic". Epidemiol Prev 2011; 3-4: 171-2.

[47] Hansson-Mild K, Hardell L, Kundi M, et al. Mobile telephones and cancer: is there really no evidence of an association? Int J Mol Med 2003; 12: 67-72.

[48] Kundi M. Mobile phone use and cancer. Occup Environ Med 2004; 61: 560-70.

[49] Kundi M, Hansson-Mild K, Hardell L, et al. Mobile telephones and cancer--a review of epidemiological evidence. J Toxicol Environ Health Crit Rev 2004; 7: 351-84.

[50] Hardell L, Carlberg M, Hansson-Mild K. Methodological aspects of epidemiological studies on the use of mobile phones and their association with brain tumors. Open Environ Sci 2008; 2: 54-61.
[51] Hardell L, Carlberg M Hansson-Mild K. Re-analysis of risk for glioma in relation to mobile telephone use: comparison with the results of the Interphone international case-control study. Int J Epidemiol 2011; 40: 1126-8.

[52] Hardell L. Wireless phone use and brain tumour risk. Eur J Oncol 2010; 5: 363-78.

[53] All the articles published in Pathophysiology 2009; 16: 67-250.

[54] All the articles published in Eur J Oncol 2010; 5: 1-403.

[55] Myung SK, Ju W, McDonnell DD, et al. Mobile phone use and risk of tumors: a meta-analysis. J Clin Oncol 2009; 27: 5565-72.

[56] International Agency for research on cancer (IARC). The Interphone Study 2010. Available from: www.iarc.fr/en/researchgroups/RAD/RC Ad.html

[57] Schuz J, Elliott P, Auvinen A, et al. An international prospective cohort study of mobile phone users and health (Cosmos): design considerations and enrolment. Cancer Epidemiol 2011; 35: 37-43.

[58] Repacholi MH, Lerchl A, Roosli M, et al. Systematic review of wireless phone use and brain cancer and other head tumors. Bioelectromagnetic 2011; doi: 10.1002/bem.20716.

(C) Levis et al.; Licensee Bentham Open.

This is an open access article licensed under the terms of the Creative Commons Attribution Non-Commercial License (http://creativecommons.org/licenses/by$\mathrm{nc} / 3.0 /$ ) which permits unrestricted, non-commercial use, distribution and reproduction in any medium, provided the work is properly cited. 